\title{
UNIVERSITYOF
}

FORWARD

THINKING

WESTMINSTER用

WestminsterResearch

http://www.westminster.ac.uk/westminsterresearch

\section{Evaluating monthly volatility forecasts using proxies at different frequencies}

Níguez, T. M.

NOTICE: this is the authors' version of a work that was accepted for publication in Finance Research Letters. Changes resulting from the publishing process, such as peer review, editing, corrections, structural formatting, and other quality control mechanisms may not be reflected in this document. Changes may have been made to this work since it was submitted for publication. A definitive version was subsequently published in Finance Research Letters, Available online 2 February 2016. DOI:

10.1016/j.frl.2016.01.008

Finance Research Letters is available online at:

https://dx.doi.org/10.1016/j.frl.2016.01.008

(C) 2016. This manuscript version is made available under the CC-BY-NC-ND 4.0 license http://creativecommons.org/licenses/by-nc-nd/4.0/

The WestminsterResearch online digital archive at the University of Westminster aims to make the research output of the University available to a wider audience. Copyright and Moral Rights remain with the authors and/or copyright owners.

Whilst further distribution of specific materials from within this archive is forbidden, you may freely distribute the URL of WestminsterResearch: ((http://westminsterresearch.wmin.ac.uk/)).

In case of abuse or copyright appearing without permission e-mail repository@westminster.ac.uk 


\title{
Evaluating Monthly Volatility Forecasts using Proxies at Different Frequencies
}

\author{
Trino-Manuel Ñ́guez* \\ Westminster Business School, University of Westminster, London (UK) \\ $\&$ \\ Monetary and Financial Studies Department, Research Division, Bank of Spain, Madrid (Spain)
}

\begin{abstract}
This paper analyses the forecastability of stock returns monthly volatility. The forecast obtained from GARCH and AGARCH models with Normal and Student's t errors are evaluated with respect to proxies for the unobserved volatility obtained through sampling at different frequencies. It is found that aggregation of daily multi-step ahead GARCH-type forecasts provide rather accurate predictions of monthly volatility.
\end{abstract}

Keywords: Data frequency; Leverage effect; Mixed data sampling; Models ranking; Volatility proxy.

JEL codes: C22; C52; C53; G32

*Department of Economics and Quantitative Methods, Westminster Business School, University of Westminster, 35 Marylebone Road, NW1 5LS London, UK. Phone: +44 (0) 227911 5000. Fax: +44 (0) 227911 5839. E-mail: T.M.Niguez@wmin.ac.uk 


\section{Introduction}

The forecast of monthly financial volatility is used in many economic and risk management decision making processes. On the one hand, macroeconomic and monetary policy decisions, relying on expectations of GDP growth and inflation targets, must take into account the financial markets volatility forecast as it closely relates to interest rates expectations (see Schwert (1989) for a comprehensive analysis on the relation between monthly stock returns volatility and macroeconomic variables). On the other hand, the forecast of long-horizon (monthly) financial volatility is key for risk management decisions, such as portfolio choice, strategic positioning, regulatory and internal capital allocation, and risk-adjusted performance measurement schemes.

The literature on modelling and forecasting volatility has undergone extensive development since the seminal autoregressive conditional heteroscedasticity $(\mathrm{ARCH})$ and generalized ARCH (GARCH) models of Engle (1982) and Bollerslev (1986). GARCH models and their generalizations, surveyed in Terasvirta (2009) and Silvennoinen and Terasvirta (2009), are currently widely used by researchers and practitioners for forecasting the dynamics of financial returns variance. The availability of high-frequency (intra-daily) financial data has motivated the development of the socalled data-driven models of realized volatility (see Andersen and Bollerslev (1998), Andersen et al. (2001, 2003), Meddahi (2002) and Barndorff-Nielsen and Shephard (2004)), and the mixed data sampling (MIDAS) regression models (Ghysels et al., 2006).

A simple method for forecasting monthly volatility consists on using returns sampled at monthly frequency to perform a 1-step-ahead GARCH-type forecast (see e.g. Schwert, 1989). This method requires a very long sample to exploit the volatility clustering feature of higher frequency returns. Alternatively, one can use daily returns and perform multi-step-ahead volatility forecasts using a GARCH-type model (see Baillie and Bollerslev, 1992). Operationally, these 1-day-ahead GARCH forecasts can be converted to longer horizons by scaling by the square root of the horizon (for 
instance, as in Morgan's (1996) RiskMetrics). Christoffersen et al. (1998) assessed these two methods and found that volatility forecastability declines quickly with the horizon vanishing beyond horizons of ten or fifteen periods (days) ahead.

In this letter, I follow the methodology based on data-driven models, to predict EuroStoxx 50 monthly volatility by aggregating multi-step (daily) ahead volatility forecasts. ${ }^{1}$ Similarly, measures of the unobservable target volatility are calculated by aggregating (future) squared or absolute returns (see Ghysels et al. (2006) and Barndorff-Nielsen and Shephard (2004)). ${ }^{2}$

The EuroStoxx 50 returns conditional variance is modeled by assuming either a GARCH model or an asymmetric GARCH (AGARCH) (Glosten et al., 1993) to account for "leverage effects", together with two distributions: the Normal distribution, and the Student's t that is flexible to account for excess kurtosis not not fully captured by Normal-GARCH processes (Bollerslev, 1987). Models are also estimated for filtered returns by outliers to eliminate the known bias in GARCH parameter estimates caused by extreme observations (Carnero et al., 2007), and forecasts based on those estimations are also analyzed.

The models performance is evaluated by using the mean squared error loss function (MSE hereafter), and the Minzer-Zarnowitz (1969) regression method.(M-Z hereafter).

The remainder of the paper is organized as follows. Section 2 presents the data and the models for conditional heteroscedasticity, and discusses the estimation results. Section 3 presents the method to measure the unobservable volatility variable. Section 4, presents the methodology and results of the monthly volatility forecasting. Finally, Section 5 summarizes the conclusions.

\footnotetext{
${ }^{1}$ Models based on monthly returns and on square-root scaling are not considered in the analysis given its known inferior performance for monthly volatility forecast. Only GARCH 1-step-ahead monthly forecasts are used as benchmark to illustrate the performance of the rest of considered models.

${ }^{2}$ Unlike most papers on volatility forecasting which focus on a horse race of different volatility models, this study investigates models performance with respect to alternative measures of the monthly volatility proxy obtained using data at different frequencies.
} 


\section{Stock returns volatility modeling}

Let daily returns be denoted by $r_{t}=\log \left(P_{t}\right)-\log \left(P_{t-1}\right)$. Throughout the paper the time index $t$ will refer to daily sampling. I also use data sampled at a lower frequency (monthly), with each month having $m$ days, then I denote the monthly return as $r_{m t}=\log \left(P_{m t}\right)-\log \left(P_{m(t-1)}\right)$. To make the analysis more realistic I consider months with their actual number of working days, so $m_{i j}$ (number of observations of month $i$ year $j$ ) is not constant and ranges from 20 to 23 days. For the sake of simplicity in notation, I drop the $i j$ subindices of $m$.

The data set used consists of daily and monthly returns of the EuroStoxx 50 index over a 19 years period, from February 1, 1988 to December 3, 2007, for a total of 5, 176 daily and 239 monthly observations. The data were downloaded from Datastream. Table 1 presents descriptive statistics of the data, which illustrate the known stylized features of financial returns: volatility clustering (Engle, 1982), heavy-tailed distribution (Mandelbrot, 1963), and asymmetric response of returns to positive and negative shocks (Black, 1976). To save space, all Figures used in this study are provided as Supplementary material. ${ }^{3}$

Table 1. Descriptive statistics of EuroStoxx 50 daily and monthly percent returns. The JarqueBera (J-B) statistic is asymptotically distributed as a $\chi^{2}$ distribution with 2 degrees of freedom under the null of normality, the p-value (p-v) of the test is in parenthesis next to the J-B statistic.

\begin{tabular}{lcccccccc}
\hline & Obs & Mean & Max & Min & St. Dev. & Skew & Kurtosis & J-B stat (p-v) \\
\hline Sample & & & \multicolumn{6}{c}{$2 / 01 / 1988-12 / 03 / 2007$} \\
Daily & 5176 & 0.0372 & 7.078 & -7.5191 & 1.1876 & -0.2228 & 7.8511 & $5118.2(0.00)$ \\
Monthly & 239 & 0.7985 & 15.306 & -20.230 & 5.1762 & -0.7252 & 4.5528 & $44.965(0.00)$ \\
\hline
\end{tabular}

Both daily and monthly returns are filtered by their conditional mean to remove small linear dependences attributed to non-synchronous trading in the stocks that form the index (see Sentana and Wadhwani, 1992). The Akaike Information Criterion (AIC hereafter) selects the following

\footnotetext{
${ }^{3}$ Figure 1 includes the plots of the daily and monthly prices and returns series.
} 
process for the conditional mean: $r_{t}=\mu+\varepsilon_{t}$; all ARMA parameters, but the intercept, were not statistically significant at least at 10 per cent level.

I assume two distributions for the returns, the Normal as benchmark and the Student's t (Bollerslev, 1987) as a more flexible distribution to fit the leptokurtosis of the data distribution. To account for volatility clustering and the "leverage effect" the conditional variance of the returns distribution is specified to follow either a $\operatorname{GARCH}(1,1)$, (eq. 2), or an $\operatorname{AGARCH}(1,1)$, eq. $3 .{ }^{4}$ To fix notation the return process is given by,

$$
\begin{aligned}
r_{t} & =\mu+\varepsilon_{t}, \\
\varepsilon_{t} & =h_{t}^{\frac{1}{2}} \eta_{t}, \quad \varepsilon_{t}\left|\Omega_{t-1} \sim N\left(0, h_{t}\right), \quad \varepsilon_{t}\right| \Omega_{t-1} \sim t_{\nu}\left(0, h_{t}\right), \\
h_{t} & =\omega+\alpha u_{t-1}^{2}+\beta h_{t-1}, \\
h_{t} & =\omega+\alpha\left(u_{t-1}-\gamma\right)^{2}+\beta h_{t-1},
\end{aligned}
$$

where $\Omega_{t-1}$ denotes the information set up to time $t-1, \nu$ is the degrees of freedom parameter of the Student's t distribution, and $h_{t}$ is the variance of the conditional distribution of $r_{t}$. For the sake of simplicity in notation, hereafter I use $r_{t}$, instead of $\varepsilon_{t}=r_{t}-\mu$, to denote filtered returns.

The models are estimated by maximum likelihood (ML) techniques and standard errors are robust Bollerslev and Wooldridge (1992). When the Normal distribution is assumed, ML estimation of GARCH and AGARCH models provides quasi-ML estimates (QMLE) which are consistent and asymptotically normal although not efficient (see Straumann and Mikosch (2006) for the analysis of the AGARCH case). Under the Student's t distribution, the GARCH models MLE are not consistent although they may be more efficient (see Newey and Steigerwald (1997) for further

\footnotetext{
${ }^{4}$ Other possibilities include APARCH (Ding et al., 1993), FIGARCH (Baillie et al., 1996), and HYGARCH (Davidson, 2004) models and their extensions. GARCH-in-Mean models (Engle et al., 1986) are not considered as they are likely to not provide significantly different performance than GARCH models for monthly volatility forecasts, since the effect of the mean factor vanishes after 2-steps-ahead volatility forecast.
} 
details).

Returns are also filtered to eliminate outliers that are known to bias the (Q)ML parameter estimates of GARCH processes (Carnero et al., 2007). In our empirical application, outliers are identified by the simple rule of 3 times the sample standard deviation $\left(3 \cdot \widehat{h}^{\frac{1}{2}}\right)$ and substituted by the returns sample mean: 117 outliers were substituted for the daily returns, and 5 for the monthly data. This filtering procedure will not affect the models ranking regarding their out-of-sample forecasting performance, since it is same for all models. ${ }^{5}$

\section{$2.1 \quad$ Estimation results}

Table 2 reports the estimation results for the models using the daily returns. Hereafter ' $n$ ' and ' $t$ ' preceded by the conditional variance process denote that the assumed distribution for the returns is either the Normal or the Student's $t$, respectively. Panel 1 presents the results for the unfiltered returns. The sum of $\widehat{\alpha}$ and $\widehat{\beta}$ is near 1 , which indicates high persistence in the EuroStoxx 50 returns daily volatility. The degrees of freedom coefficient, $\widehat{\nu}$, is around 6 , confirming the existence of leptokurtosis in the returns distribution. The asymmetry parameter estimate, $\hat{\gamma}$, is statistically different from zero or one, confirming the existence of the "leverage effect" in the index return daily volatility.

The estimation results in Panel 2 for the filtered returns show that the returns distribution is nearer to normality ( $\widehat{\nu}$ is higher for the unfiltered returns). Furthermore, we note that the bias of $\widehat{\alpha}$ and $\widehat{\beta}$ is not corrected for the first sample window, although careful monitoring of estimation reveals that it does correct for other windows over the out-of-sample period, for which $\widehat{\nu}$ take values above 25. According to the AIC, asymmetry and Student's t errors both provide the GARCH specification

\footnotetext{
${ }^{5}$ Alternative procedures, applicable in a forecasting volatility context, to correct for outliers in returns series with GARCH effects are proposed in Franses and Ghysels (1999).
} 
with more flexibility to fit the data.

Table 2. Models estimation results for daily returns across the in-sample period $(2 / 01 / 1988$ 1/01/1999, observations 2,850). (Q)MLE estimates, and t-statistics (in parenthesis) obtained from robust standard errors. Panel 1 presents the model estimation for the original sample, and Panel 2 for the sample corrected by substituting outliers (returns larger than 3 times the sample standard deviations) by the returns sample mean.

\begin{tabular}{lcccc}
\hline & GARCH-n & AGARCH-n & GARCH-t & AGARCH-t \\
Panel 1: No Filter & & & & \\
$\omega$ & $.0475(2.48)$ & $.0279(2.11)$ & $.0226(3.65)$ & $.0189(3.25)$ \\
$\alpha$ & $.1113(4.47)$ & $.0889(3.36)$ & $.0226(5.68)$ & $.0934(5.95)$ \\
$\beta$ & $.8370(26.7)$ & $.8616(24.2)$ & $.8797(44.5)$ & $.8789(45.5)$ \\
$\gamma$ & & $.4445(3.08)$ & & $.2661(4.10)$ \\
$\nu$ & & & $6.037(7.47)$ & $6.207(7.33)$ \\
LogL & -3641.9 & -3623.9 & -3483.5 & -3476.1 \\
AIC & 2.5578 & 2.5459 & 2.4474 & 2.4428 \\
Panel 2: Filter $3 * \widehat{h}^{\frac{1}{2}}$ & & & & \\
$\omega$ & $.0110(2.78)$ & $.0073(1.93)$ & $.0085(2.48)$ & $.0062(1.91)$ \\
$\alpha$ & $.0607(5.43)$ & $.0567(5.51)$ & $.0617(5.17)$ & $.0612(5.43)$ \\
$\beta$ & $.9238(63.1)$ & $.9284(67.8)$ & $.9275(62.9)$ & $.9272(65.1)$ \\
$\gamma$ & & $.2625(5.40)$ & & $.2375(3.56)$ \\
$\nu$ & & & $9.333(6.23)$ & $9.7201(5.93)$ \\
LogL & -3383.4 & -3374.8 & -3356.8 & -3350.6 \\
AIC & 2.3764 & 2.3711 & 2.3584 & 2.3548 \\
\hline
\end{tabular}

\section{The volatility proxy}

The goal is to predict a measure of volatility over a monthly horizon that corresponds to: 1step-ahead forecast for monthly frequency data, $\widetilde{h}_{m(t+1)}$, and to $m$-days-ahead forecast for daily frequency, $\widetilde{h}_{t+m}$. As volatility is an unobservable variable, and in order to make the analysis comparable to a large body of existing literature, I consider several measures of volatility for the period $t$ to $t+m$ (daily frequency), or $m t$ to $m(t+1)$ (monthly frequency). As primary measures, I consider the squared and absolute value monthly returns, denoted as $r_{m(t+1)}^{2}$ and $\left|r_{m(t+1)}\right|$, respectively. Following the volatility literature on high-frequency (intra-daily) data, I also consider the increments in the quadratic variation, $H_{t+m}$, (see Ghysels et al., 2006) and the "realized power" variation, $P_{t+m}$, of the return process (see Barndorff-Nielsen and Shephard, 2004). Those 
variables are not observed directly but can be measured with some discretization error through: the sum of future (a) daily squared and (b) absolute value returns, denoted as $\widetilde{H}_{t+m}=\sum_{j=1}^{m}\left(r_{t+j}\right)^{2}$ and $\widetilde{P}_{t+m}=\sum_{j=1}^{m}\left|r_{t+j}\right|$, respectively. Note that I aggregate daily returns to obtain measures of "realized" monthly volatility since the target is the forecast of monthly volatility. ${ }^{6}$

Figure 2 presents the four different EuroStoxx 50 returns monthly volatility measures over the out-of-sample period for unfiltered (Panel 1) and filtered returns (Panel 2). It is observed that monthly volatility tends to be underestimated when using punctual monthly squared returns in relation to the aggregated measure of quadratic variation. This underestimation is systemic when considering punctual monthly absolute value returns in relation to the "realized power" volatility.

It is worth noting that, in the case of discrete time processes, the proxies $r_{m(t+1), m t}^{2}$ and $\widetilde{H}_{t+m, t}=\sum_{j=1}^{m}\left(r_{t+j, t}\right)^{2}$ are unbiased measures for the implied (true) underlying monthly volatility, and although the former is noisier, both ensure a correct ranking of models, provided that the loss function is quadratic (see e.g. Andersen and Bollerslev (1998), Awartani and Corradi (2005) and Hansen and Lunde (2005)).

\section{Volatility forecasting methodology}

For the monthly forecasts using the monthly frequency, I use the first 132 observations to estimate the parameters of the GARCH models, and compute $N=107$ out-of-sample (February 1999 to December 2007) 1-step-ahead forecasts of the conditional variance, $\mathbf{E}_{m t}\left(r_{m(t+1)}^{2}\right)=\widehat{h}_{m(t+1)}$, by using a rolling window of constant size $239-N$ that discards old observations.

For the daily frequency, I use the first 2,850 observations to estimate the parameters of the GARCH models, and compute $N=107$ out-of-sample $m$-step-ahead forecasts of the conditional

\footnotetext{
${ }^{6}$ Higher frequency data (intra-daily) could be used for the monthly proxy, but this option is discarded since for monthly-horizon forecasts there are not significant differences in performance, as shown in Ghysels et al. (2006)
} 
variance by using a rolling window of non-constant size $5,176-(N * m)$ that discards the $m$ oldest observations and incorporates the newest $m$ observations of the month that just went; the out-ofsample period is February 2, 1999 to December 3, 2007 (2,326 daily observations corresponding to 107 months $).{ }^{7}$ Then I use a recursive multi-step-ahead forecasting procedure (Baillie and Bollerslev, 1992) where the optimal predictor for the 1 -step-ahead $\operatorname{GARCH}(1,1)$ conditional variance is given by

$$
\widehat{h}_{t+1} \equiv E_{t}\left(h_{t+1}\right)=\omega+\alpha u_{t}^{2}+\beta h_{t}
$$

and the $m$-step-ahead optimal predictor is

$$
\widehat{h}_{t+m} \equiv E_{t}\left(h_{t+m}\right)=\omega+(\alpha+\beta) h_{t+m-1} .
$$

For the AGARCH$(1,1)$, the optimal $m$-step-ahead predictor of the conditional variance is given by,

$$
E_{t}\left(h_{t+m}\right)= \begin{cases}\omega+\alpha\left(u_{t}-\gamma\right)^{2}+\beta h_{t}, & \text { for } m=1, \\ \omega+(\alpha+\beta) h_{t+m-1}+\alpha \gamma^{2}, & \text { for } m>1\end{cases}
$$

Then, a monthly conditional variance forecast is obtained by adding the previous $m$-steps-ahead conditional variance forecasts, $\widehat{H}_{t+m}=\sum_{t+j}^{m} \widehat{h}_{t+j}$. When the target volatility is either $\widetilde{P}_{t+m}$ or $\left|r_{m(t+1)}\right|$, the monthly forecast predictor is: $\widehat{H}_{t+m}^{\frac{1}{2}}=\sum_{t+j}^{m} \widehat{h}_{t+j}^{\frac{1}{2}}$. In summary, this procedure simulates realistic monthly forecasts of EuroStoxx 50 volatility from February 1999 to December 2007, performed the first day of each month over that period, with an updating window that discards the oldest month observations and incorporates the daily data from the latest month.

The models performance is measured by using the MSE with respect to the volatility proxies described in Section 2, namely $\widetilde{H}_{t+m}, \widetilde{P}_{t+m}, r_{m(t+1)}^{2}$ and $\left|r_{m(t+1)}\right|$. MSE are calculated for filtered (returns series free of outliers) and unfiltered returns. The volatility models forecasting performance obtained by using the filtered returns is measured with respect to either filtered or unfiltered volatility proxies. I also consider the M-Z regression to measure the models forecasting performance.

\footnotetext{
${ }^{7}$ Note that in this case window sizes may differ at most in 3 daily observations, a small number given the window size, but all windows have the same number of months.
} 
It consists on estimating the following equation,

$$
\widetilde{H}_{t+m}=\vartheta_{0}+\vartheta_{1} \widehat{H}_{t+m}+u_{t+m} .
$$

Thus, the forecast from a model is optimal with respect to the available information set $\left(\Omega_{T+i-1}\right)$ if the null $H_{0}:\left(\vartheta_{0}, \vartheta_{1}\right)=(0,1)$ is accepted. ${ }^{8}$

\subsection{Forecast results}

Figure 3 presents the plots of the monthly volatility forecasts, $\widehat{H}_{t+m}$, obtained from a GARCH- $n$, and punctual 1-step-ahead monthly forecasts obtained from a GARCH-n model fitted to monthly data, with respect to proxies $\widetilde{H}_{t+m}$ and $r_{m(t+1)}^{2}$. It is clearly observed that $\widehat{H}_{t+m}$ is much more flexible to capture periods of high volatility in relation to both proxies and, both methods (aggregation and monthly punctual forecasts) provide similar results for periods of low volatility.

Figure 4 presents plots of $\widehat{H}_{t+m}$ obtained from AGARCH- $n$ and AGARCH- $t$ models against the proxy $\widetilde{H}_{t+m}$. From the plots it can be observed that both models provide reasonably good forecasts being difficult to discriminate between them.

Figure 5 presents monthly forecasts $\widehat{H}_{t+m}$ and $\widehat{H}_{t+m}^{\frac{1}{2}}$, from a GARCH- $n$ model and filtered returns against (filtered and unfiltered) proxies $\widetilde{H}_{t+m}$ and $\widetilde{P}_{t+m}$, respectively. As expected, it is observed a better fit of forecasts $\widehat{H}_{t+m}$ to the proxy $\widetilde{H}_{t+m}$ than when unfiltered data are considered. But $\widehat{H}_{t+m}$ obtained from filtered data are not able to capture "real" periods of high volatility, as shown by the large discrepancy between $\widehat{H}_{t+m}$ and $\widehat{H}_{t+m}^{\frac{1}{2}}$ from filtered data and proxies $\widetilde{H}_{t+m}$ and $\widetilde{P}_{t+m}$ from non-filtered returns. Note that $\widetilde{H}_{t+m}$ and $\widetilde{P}_{t+m}$ for non-filtered returns are the actual (observed) proxies of volatility and so the target variables.

A sharp result that emerges from Table 3 (below) is that, for both filtered and unfiltered returns, Normal models provide a lower MSE than their Student's t counterparts, being the AGARCH- $n$

\footnotetext{
${ }^{8}$ Note that, without loss of generality, the M-Z equation is specified for the proxy $\widetilde{H}_{t+m}$ and the forecasts $\widehat{H}_{t+m}$.
} 
generally preferred to the GARCH- $n$ model. This result is consistent with those in the existing literature that show that the heavy-tail assumption in GARCH models helps to better forecast measures such as value-at-risk rather than conditional variance (see, e.g., Brooks and Persand (2003), Awartani and Corradi (2005), and Níguez (2008)). In relation to the M-Z regression criteria, it is worth noting that the null of optimal forecasts is accepted only for Normal models when the target variable is $\widetilde{H}_{t+m}$, being rejected for the rest of cases at any reasonable significance level. It stands out the high $R^{2}$ found from models when either $\widetilde{H}_{t+m}$ and $\widetilde{P}_{t+m}$ are used as proxies; these values are higher than those generally found in the literature. On the other hand, when proxies $r_{m(t+1)}^{2}$ and $\left|r_{m(t+1)}\right|$ are used (see Table 4) the values reported are in line with those in the literature (see, for instance, Andersen and Bollerslev, 1998). Furthermore, $R^{2}$ from models using filtered data are higher, as expected. It is also worth mentioning that for unfiltered returns the same model ranking is found with respect the MSE when using either $\widetilde{H}_{t+m}$ or $r_{m(t+1)}^{2}$ as proxies for the implied (underlying) volatility.

Table 3. Out-of-sample monthly forecasting performance with respect to quadratic variation (Panel 1) and absolute value variation (Panel 2) proxies, for filter and unfiltered returns. Predictions are obtained from GARCH models fitted to daily filtered and unfiltered returns. P-value and $R^{2}$ from the Minzer-Zarnowitz regression, and MSE stands for Mean Square Error.

\begin{tabular}{lcccc}
\hline & GARCH-n & AGARCH-n & GARCH-t & AGARCH-t \\
Panel 1: Proxy $\widetilde{H}_{t}$ & & & & \\
No Filter & & & & \\
P-value & 0.0726 & 0.1601 & 0.0071 & 0.0306 \\
$R^{2}$ & 0.4726 & 0.4832 & 0.4809 & 0.4904 \\
MSE & 1566.9 & 1512.4 & 1612.4 & 1539.3 \\
Filter $3^{*} \widehat{h}^{\frac{1}{2}}$ & & & & \\
P-value & 0.2287 & 0.1816 & 0.0369 & 0.0142 \\
$R^{2}$ & 0.3816 & 0.4022 & 0.3927 & 0.4118 \\
MSE & 147.3 & 143.0 & 149.7 & 147.7 \\
Panel 2: Proxy $\widetilde{P}_{t}$ & & & & \\
No Filter & & & & \\
P-value & 0.0000 & 0.0000 & 0.0000 & 0.0000 \\
$R^{2}$ & 0.5448 & 0.5622 & 0.5621 & 0.5754 \\
MSE & 99.3 & 96.4 & 111.3 & 106.2 \\
Filter 3* $\widehat{h}^{\frac{1}{2}}$ & & & & \\
P-value & 0.0000 & 0.0000 & 0.0000 & 0.0000 \\
$R^{2}$ & 0.3602 & 0.3793 & 0.3724 & 0.3791 \\
MSE & 49.3 & 51.3 & 54.7 & 56.9 \\
\hline
\end{tabular}


Table 4. Out-of-sample monthly forecasting performance with respect to squared (Panel 1) absolute value (Panel 2) monthly returns. Predictions are produced by using GARCH models fitted to daily filtered and unfiltered returns. P-value and $R^{2}$ from Minzer-Zarnowitz regression, and MSE stands for Mean Square Error.

\begin{tabular}{lcccc}
\hline & GARCH-n & AGARCH-n & GARCH-t & AGARCH-t \\
Panel 1: Proxy monthly $r_{m t}^{2}$ & & & & \\
No Filter & & & & \\
P-value & 0.0000 & 0.0000 & 0.0000 & 0.0000 \\
$R^{2}$ & 0.1239 & 0.1232 & 0.1322 & 0.1304 \\
MSE & 3495.8 & 3435.6 & 3730.5 & 3636.9 \\
Filter $3^{*} \widehat{h}^{\frac{1}{2}}$ & & & & \\
P-value & 0.0000 & 0.0000 & 0.0000 & 0.0002 \\
$R^{2}$ & 0.3892 & 0.3670 & 0.3818 & 0.3577 \\
MSE & 550.6 & 547.1 & 522.6 & 524.1 \\
Panel 2: Proxy monthly $\left|r_{m t}\right|$ & & & & \\
No Filter & & & & \\
P-value & 0.0000 & 0.0000 & 0.0000 & 0.0000 \\
$R^{2}$ & 0.1548 & 0.1558 & 0.1632 & 0.1646 \\
MSE & 617.6 & 617.8 & 672.7 & 665.0 \\
Filter $3^{*} \widehat{h}^{\frac{1}{2}}$ & & & & \\
P-value & 0.0000 & 0.0000 & 0.0000 & 0.0000 \\
$R^{2}$ & 0.4588 & 0.4295 & 0.4593 & 0.4281 \\
MSE & 315.0 & 323.6 & 332.8 & 341.6 \\
\hline
\end{tabular}

\section{Conclusions}

This article provides a study of the predictability of stocks monthly volatility. I consider different measures for the unobservable target monthly volatility including, monthly squared returns and an estimate of the increments of the returns quadratic (absolute value) variation calculated using daily future squared (absolute value) returns.

I analyse the forecasting performance of GARCH and AGARCH models with Normal and Student's t errors together with a procedure that aggregates Baillie and Bollerslev's (1992) multistep-ahead volatility optimal forecasts to predict monthly volatility. I find that this method provides rather accurate results of monthly volatility in relation to other methods based on either 1-stepahead GARCH-type forecasts using monthly frequency, or multi-step-ahead (without aggregation) GARCH-type forecasts using daily returns (see Christoffersen et al. (1998)). Normal AGARCH models seem to provide more accurate volatility forecasts according to the MSE loss functions and M-Z regression criteria. 


\section{References}

[1] Andersen, T.G., Bollerslev, T. (1998) Answering the skeptics: Yes, standard volatility models do provide accurate forecasts. International Economic Review 39, 885-905.

[2] Andersen, T.G., Bollerslev, T., Diebold, F.X., Ebens, H. (2001) The distribution of realized stock return volatility. Journal of Financial Economics 61, 43-76.

[3] Andersen, T.G., Bollerslev, T., Diebold, F.X., Labys, P. (2003) Modelling and forecasting realized volatility. Econometrica 71, 579-625.

[4] Awartani, B.M.A., Corradi, V. (2005) Predicting the volatility of the S\&P-500 stock index via GARCH models: the role os asymmetries. International Journal of Forecasting 21, 167-183.

[5] Baillie, R.T., Bollerslev, T., Mikkelsen, H. (1996) Fractionally integrated generalized autoregressive conditional heteroscedasticity. Journal of Econometrics 74, 3-30.

[6] Barndorff-Nielsen, O.E., Shephard, N. (2001) Non Gaussian OU based models and some of their use in financial economics. Journal of the Royal Statistical Society 63, 167-207.

[7] Black, F. (1976) Studies of stock price volatility changes. Proceedings of the 1976 Meetings of the American Statistical Association, Business and Economic Statistics Section, 177-181.

[8] Bollerslev, T. (1986) Generalized autoregressive conditional heteroscedasticity. Journal of Econometrics 31, 307-327.

[9] Bollerslev, T. (1987) A conditional heteroskedastic time series model for speculative prices and rates of return. Review of Economics and Statistics 69, 542-547.

[10] Bollerslev, T., Wooldridge, J.M. (1992) Quasi-Maximum likelihood estimation and inference in dynamics models with time-varying covariances. Econometrics Reviews 11, 143-172.

[11] Brooks, C., Persand, G. (2003) The effect of asymmetries on stock index return value-at-risk estimates. Journal of Risk Finance 4, 29-42. 
[12] Carnero, M.A., Peña, D., Ruiz, E. (2007) Effects of outliers on the identification and estimation of GARCH models. Journal of Time Series Analysis 4, 471-497.

[13] Christoffersen, P.F., Diebold, F.X., Schuermann, T. (1998) Horizon problems and extreme events in financial risk management. FRBNY Economic Policy Review, October, 109-118.

[14] Davidson, J. (2004). Moment and memory properties of linear conditional heteroscedasticity models, and a new model. Journal of Business and Economic Statistics 22, 16-29.

[15] Ding, Z., Granger, C. W. J., and Engle, R. F. 1993. A long memory property of stock market returns and a new model. Journal of Empirical Finance 1, 83-106.

[16] Engle, R.F. (1982) Autoregressive conditional heteroscedasticity with estimates of the variance of U. K. inflation. Econometrica 50, 987-1008.

[17] Engle, R.F., Lilien, D.M., Robins R.P. (1987). Estimating time varying risk premia in the term structure: The ARCH-M model. Econometrica 55, 391-407.

[18] Franses, P.H., Ghysels, E. (1999). Additive outliers, GARCH and forecasting volatility. International Journal of Forecasting 15, 1-9.

[19] Ghysels, E., Santa-Clara, P., Valkanov, R. (2006) Predicting volatility: getting the most out of return data sampled at different frequencies. Journal of Econometrics 131, 59-95.

[20] Glosten, L.R., Jagannathan, R., Runkle, D. (1993) On the relation between the expected value and the volatility of the nominal excess return on stocks. Journal of Finance 48, 1779-1801.

[21] Hansen, P.R., Lunde, A., (2005) A forecast comparison of volatility models: does anything beat a GARCH(1,1)? Journal of Applied Econometrics 7, 873-889.

[22] Mandelbrot, B. (1963) The variation of certain speculative prices. Journal of Business 36, 1279-1313. 
[23] Meddahi, N. (2002) A theoretical comparison between integrated and realized volatilities. Journal of Applied Econometrics 17, 479-508.

[24] Mincer, J., Zarnowitz, V. (1969) The evaluation of economic forecasts. In Economic forecasts and expectations, J. Mincer (ed.), . New York: NBER.

[25] Morgan, J.P. (1996) RiskMetrics-Technical Document (4th ed.). Morgan Guaranty Trust Company: New York.

[26] Newey, W., Steigerwald, D. (1997) Asymptotic bias for quai-maximum-likelihood estimators in conditional heteroskedasticity models. Econometrica 65, 587-599.

[27] Ñ́guez, T.M. (2008). Forecasting volatility in the Madrid Stock Exchange. Spanish Economic Review 10, 169-196.

[28] Schwert, G.W. (1989) Why does stock market volatility change over time? Journal of Finance $5,1115-1153$.

[29] Sentana, E., Wadhwani, S. (1992) Feedback traders and stock return autocorrelations: Evidence from a century of daily data. Economic Journal 102, 415-425.

[30] Straumann, D., Mikosch, T. (2006) Quasi-maximum-likelihood estimation in conditionally heteroscedastic time series: A stochastic recurrence equations approach. Annals of Statistics $34,2449-2495$.

[31] Silvennoinen, A., and Terasvirta, T. (2009). Multivariate GARCH models. In Handbook of Financial Time Series, T. G. Andersen, R. A. Davis, J.-P. Kreiß, and T. Mikosch, (eds.). New York: Springer.

[32] Terasvirta, T. (2009). An introduction to univariate GARCH models. In Handbook of Financial Time Series, T. G. Andersen, R. A. Davis, J.-P. Kreiß, and T. Mikosch, (eds.). New York: Springer. 